\title{
Shell effects in alkali metal nanowires
}

\author{
A. I. Yanson ${ }^{1,2}$, I. K. Yanson ${ }^{1,3}$, and J. M. van Ruitenbeek ${ }^{1}$ \\ 1 Kamerlingh Onnes Laboratorium, Leiden University, PO Box 9504, NL-2300 RA Leiden, The Netherlands \\ 2 Present address: Dept. of Physics, 510 Clark Hall, Cornell University, Ithaca, NY 14853 \\ 3 B. Verkin Institute for Low Temperature Physics and Engineering of the National Academy of Sciences \\ 47 Lenin ave., 61103, Kharkov, Ukraine
}

Received July 3, 2001

\begin{abstract}
After making a cold weld by pressing two clean metal surfaces together, and gradually separating the two pieces, a metallic nanowire is formed, which is progressively thinning down to a single atom before the contact is lost. We show evidence that the radii of such nanowires are influenced by electronic shell filling effects, in analogy to electronic shell structure in metal clusters. A modulation of the shell effect, known as supershell structure, is clearly observed. For sodium and potassium at larger diameters there is a crossover to crystalline facets with shell-closings corresponding to the completion of an additional atomic layer. We conclude that a complete analogy exists between shell structure in metallic clusters and nanowires.
\end{abstract}

PACS: 73.20.Dx, 73.40.Jn

\section{Introduction}

The understanding of processes that occur on an atomic scale when two materials are brought into contact is of fundamental importance to problems such as adhesion, hardness, plastic and elastic response to external strength, fracture, friction and wear [1]. For conducting materials a new phenomenon appears due to quantization of conduction channels [2-4]. Novel techniques enable one to create and study the properties of nanowires down to a chain of single atoms $[5,6]$. For these experiments, as well as for the above-mentioned problems, the processes connected with stability and cohesive energy of nanoscopic contacts are of primary importance.

The stability of microscopic and nanoscopic objects is tightly connected with the so-called shell effects. In three dimensions, shell effects are concerned with the numbers of microscopic particles (barions in nuclei, electrons in an atom, atoms in a cluster) for which an enhanced stability of the system is found. These are called «magic numbers». For the periodic table of elements the noble gases, with fully occupied electronic shells, acquire the highest stability, since they possess the largest ionization potential and are chemically inert. For the clusters emitted by a vapor jet in a mass spectrometer, the abundance of «magic masses» is enhanced, since these clusters possess higher stability as compared to the others [7-9].

In metallic clusters, one distinguishes two different shell effects: one is connected with conduction electrons for which the cluster behaves like a giant atom [7-9], and the other relates to the configuration of atoms in the cluster $[9,10]$. Both are due to minima in the cluster free energy, but the first requires conduction electrons, while the second also applies to non-conducting materials.

In this paper we describe in detail the shell effects discovered by us in an open system: a metallic nanowire [11-13]. In these objects electrons are not confined along the wire axis. In spite of this, shell effects manifest themselves as peaks in a histogram for the electrical conductance of the wire recorded for wires while the wire diameters are changed, showing the most stable configurations. Since the conductance depends on the wire crosssectional area, the latter quantity gives, on the one hand, the number of conduction electron modes in the two lateral dimensions, and, on the other hand, the number of atoms. Both of these effects, electronic and atomic, are distinctly seen in the conductance histograms, which exhibit their coexistence and crossover. Thus, there appears to be a complete analogy between the shell effects in metallic clusters and nanowires. 


\section{Experimental technique}

For our study of nanowires we use (Fig. 1,a) the mechanically controllable break-junction (MCB) technique developed in Ref. 14. The metallic sample was fixed by Stycast epoxy on a flexible substrate made from phosphor bronze (bending beam) and supported by three points: two are on the upper side of the bending beam (counter supports), and the third is at the opposite side supported by a movable piezo driver. A metallic sample, prepared as a thick wire, is electrically isolated from the bronze substrate. The wire was circularly notched in the middle in order to concentrate the pulling strength at the center while the substrate was bent by mechanical (not shown) and piezo drivers. By fixing the sample as close as possible near the notch, a large reduction $(100-1000)$ is achieved in the ratio between movement of the electrodes with respect to each other and the expansion of the piezo driver, in the perpendicular direction.

In order to study alkali metals, two important modifications are made. First, due to extraordinary reactivity of alkaline elements contact with the ambient environment must be avoided. This is achieved by making all the preparations in paraffin oil. The sample is cut into the shape of a rectangular bar, at the center of which we cut a notch. Since the bar with the notch is also covered by the oil, it cannot be glued and should be fixed mechanically. For this purpose we use four tiny bolts and nuts that are electrically isolated from the substrate (Fig. 1,b) [4]. After mounting, the whole setup is rapidly placed in an evacuated metallic can and put into the cryostat with an environmental temperature of $4.2 \mathrm{~K}$. The walls of the can that are being kept at helium temperature serve as a cryogenic pump ensuring a very high vacuum around the sample. The bar is then broken at the notch by mechanical bending of the substrate. The cyclic electrical movement of the piezo drive rod periodically sweeps the electrical conductance between the electrodes from a desired value ( of the order of tens to hundreds of conductance quanta ${ }^{*}$ ) down to a complete separation (conductance much less than one quantum unit). The important advantage of the MCB device is that the cryopumping action of the low-temperature environment ensures that the freshly exposed fracture surfaces are not polluted by adsorbates.

The conductance is measured in a four-terminal dc constant voltage mode. A fixed bias voltage is applied, in the range $1-100 \mathrm{mV}$, so that the current

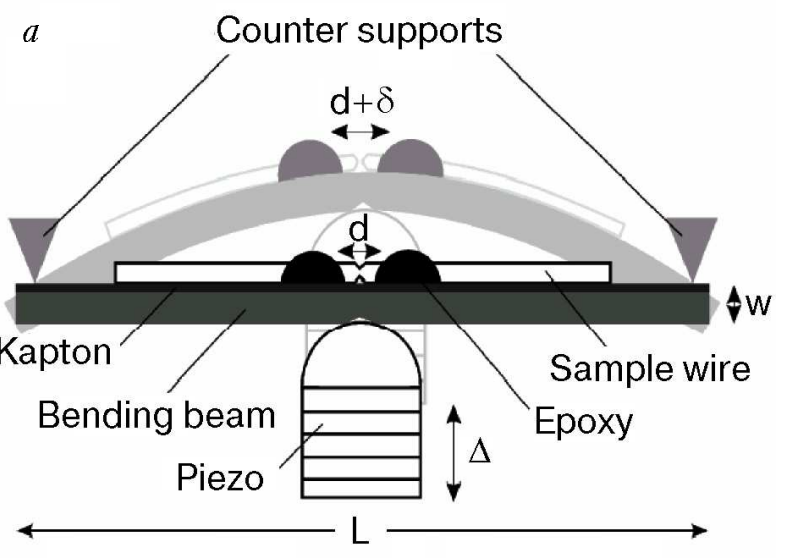

$b$

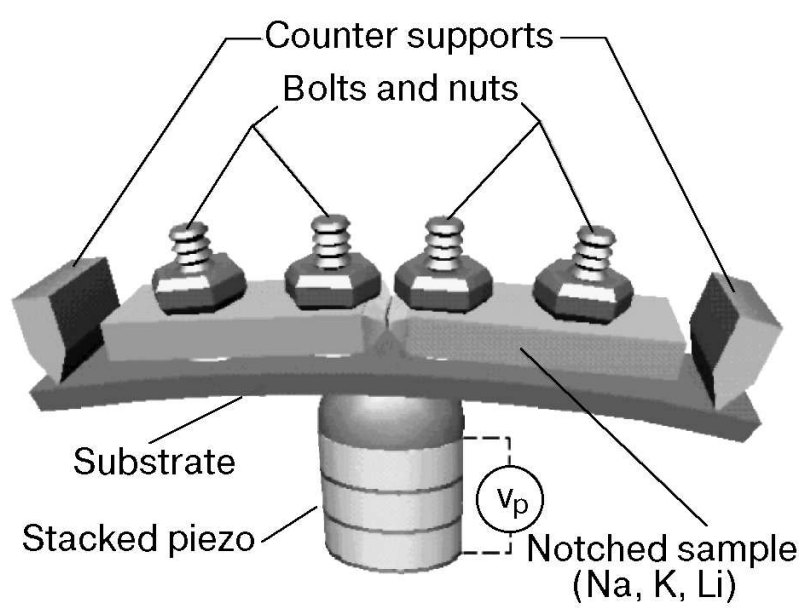

Fig. 1. $a-$ Schematic drawing of a substrate with a sample mounted in a three-point bending configuration. The bending of the substrate due to the movement (or expansion) of the piezo over a distance $\Delta$, shown in gray, causes the epoxy droplets to move apart by $\delta$, leading to the breaking of the junction. The amount of bending is exaggerated for clarity. $b-\mathrm{A}$ model of an alkali break junction. The principle is the same here as in $a$, but the two epoxy drops are replaced by four M1 bolts with nuts fixing the sample to the substrate. The length of the substrate remains $22 \mathrm{~mm}$.

through the contact is proportional to its conductance. Since the high bias heats the wire at large conductances, we prefer to use the smallest possible biases. Our experience leads to the conclusion that up to the bias of $\sim 10 \mathrm{mV}$ the heating can be neglected. The drift and calibration of the currentto-voltage converter is verified against standard conductors of 1,10 and $100 G_{0}$, ensuring an overall accuracy in the conductance better than $1 \%$ for $G>10 G_{0}$. Scans are taken continuously by ramping the displacement $\delta \equiv l$ of the electrodes with

* One conductance quantum equals $G_{0} \equiv 2 e^{2} / h=(12907 \Omega)^{-1}$. 
respect to each other, using the piezo driver. Each individual curve of the conductance versus the displacement, $G(l)$, is recorded in $\sim 0.1$ seconds from the highest conductance into the tunneling regime. The curves are digitized with 16-bit resolution and a data acquisition rate of $\sim 10^{5}$ point $/ \mathrm{s}$. The conductance scale is divided into 1500 bins and data points from up to $\sim 10^{5}$ individual scans are accumulated automatically, creating a conductance histogram at the given experimental conditions (temperature, voltage bias, indentation depth). For most cases we have found that about $10^{3}$ scans are enough to achieve convergence in the shape of the histograms. The speed of scanning is limited from above by the data acquisition rate of the electronics, and from below by a reasonable time required for the experiment (normally, several hours). To compare different histograms, we normalized them by their area over a fixed conductance range.

\section{Results}

\subsection{Experiment}

Low temperature histograms and scans. In Fig. 2 we show histograms for $\mathrm{Na}, \mathrm{K}$ and $\mathrm{Li}$ taken at $T=4.2 \mathrm{~K}$, along with typical scans (see inset). All histograms display the characteristic series of peaks in succession at $1,3,5,6$, in units of reduced conductance ${ }^{*}$ which was shown in theoretical and experimental works $[4,15,16]$ to be the hallmark of quantum mode degeneracy for electronic wave functions in a wave guide with circular cross section. This degeneracy arises from the azimuthal quantum number, which takes two values $(-$ and +$)$. When this happens (at $g=1,3$, and 6 , and further on) the conductance increases by 2 quanta for increasing nanowire diameter.

First we discuss the positions of these peaks. They are more or less shifted to the left compared to the integer values. The least shift occurs for the $g=1$ peak and it increases noticeably for higher $g$. Phenomenologically, this increase can be described by a series resistance of the order of $\sim 100 \Omega$, originating from the backscattering of electron wave functions by defects and surface irregularities $[4,17,18]$, and inelastic scattering by phonons. The latter may be the cause for a noticeably larger shift for $\mathrm{Li}$, for which it is known the electron-phonon interaction parameter is several times larger than for $\mathrm{Na}$ and K [19].

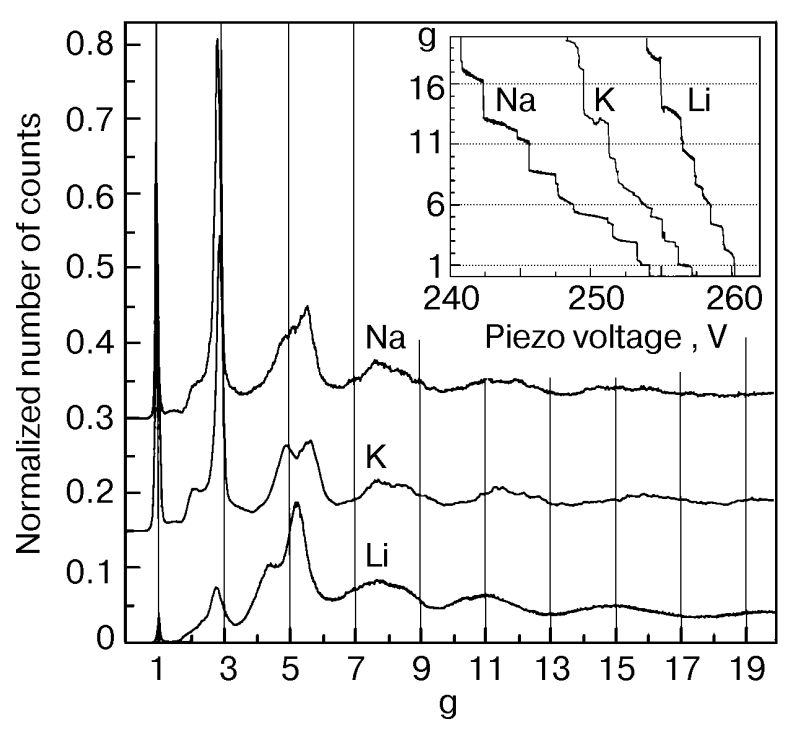

Fig. 2. Normalized conductance histograms of $>1000$ individual conductance traces of $\mathrm{Na}, \mathrm{K}$ and $\mathrm{Li}$ measured at $4.2 \mathrm{~K}$ under cryogenic vacuum at a constant voltage bias of $10 \mathrm{mV}$. The curves for $\mathrm{K}$ and $\mathrm{Na}$ are shifted for clarity by 0.15 and 0.3 , respectively. Typical individual conductance traces (scans) are shown in the inset. $g=G / G_{0}$, where $G_{0}=2 e^{2} / h$, is the reduced conductance.

The intensities of the first few peaks for $\mathrm{Na}$ and $\mathrm{K}$, on the one hand, and $\mathrm{Li}$ on the other hand, are also quite different. The height of the peaks at $g=1$ and 3 in $\mathrm{Li}$ is much lower than for $\mathrm{Na}$ and $\mathrm{K}$. On the contrary, the peaks at $g=5,6$ in $\mathrm{Li}$ is noticeably higher than those for $\mathrm{Na}$ and $\mathrm{K}$. This may be explained qualitatively by a much higher mobility of $\mathrm{Li}$ atoms even at helium temperature, which may be due to tunneling because of the light $\mathrm{Li}$ mass and the decrease in the potential barriers at the surface, making a contact of just a few atoms unstable.

Next, we draw attention to the broad maxima at $g \approx 8,11,15$, which are slightly more intense just in $\mathrm{Li}$, presumably, again due to the enhanced mobility of $\mathrm{Li}$ surface atoms. We shall see below that these broad peaks are precursors of the electronic shell-effect for alkali metal nanowires. Together with the peak at $g=5$ they give the most favorable diameters, corresponding to the local minima in the electronic part of the thermodynamic potential. As to peak at $g=5$, this point of view is supported taking into account that, according to the theoretical calculation of sodium histogram [20], the intensity of the $g=5$ peak should be noticeably smaller than the peak at $g=6$. In fact, in experimental histograms (see Fig. 2) they are approximately of

* From now on we shall use the reduced conductance as $g=G / G_{0}$, where $G_{0}=2 e^{2} / h$. 
the same intensity. Another confirmation that the $g=5$ peak is not connected with the fully open conducting channel comes from Ref. 18, where its behavior is radically different from that of the peaks at $g=1,3,6$. Thus, we propose that the enhanced intensity of the $g=5$ peak is due to the broad maximum of the favorable diameters dictated by the electronic shell effect.

Scans taken at helium temperature and shown in the inset in Fig. 2, exhibit smooth variations (plateaus) interrupted by a sharp decrease (steps), evidencing that the elastic elongations are followed by atomic rearrangements. This points at the crystalline structure of the neck. Only for the low-lying conductances the plateaus coincide with quantum units $(\approx 1,3,5,6)$, which can be even seen statistically on the average scan plotted by integrating the histogram. For higher conductance, $g \gtrsim 7$, though the steps in the scans look as sharp as for lower $g$, no peaks on the low-temperature histogram appear. This means that the plateaus occur with nearly equal probabilities for any conductances in different scans, leading on the average to the smooth background in the histograms. According to Ref. 21, from the smooth part of the scans one can find the approximate shape of the neck. We shall turn to this issue below when discussing the atomic shell structure of the nanowires. At low temperatures, the piezo voltage versus elongation of the neck can be calibrated using the Fowler-Nordheim formula or the Gundlach oscillations [22] for the tunnel regime at biases of the order of a few Volt.

By storing several intermediate histograms during the accumulation of the final histograms, for the full number of scans for a given contact, one can obtain an impression of their evolution with increasing numbers of scans. One can observe both the purification of the metal under the contact, which leads to more distinct shell-effect structure with increasing number of indentations, as well as to the opposite behavior, which we ascribe to the adsorbed impurities [23].

Temperature dependence of the scans. Let us describe the temperature dependence of the scans, taking $\mathrm{Li}$ as example (Fig. 3). Figure 3, $a$ shows two scans for low and elevated temperatures, typical for all the alkali metals studied. As the temperature increases, the low-lying conductance plateaus become unstable. Moreover, the piezo voltage is no longer the only parameter controlling the evolution of the neck, as a result of the strongly enhanced diffusion of the surface atoms. The atoms in the wire experience tension not only from the external force but also from the Laplace pressure. This
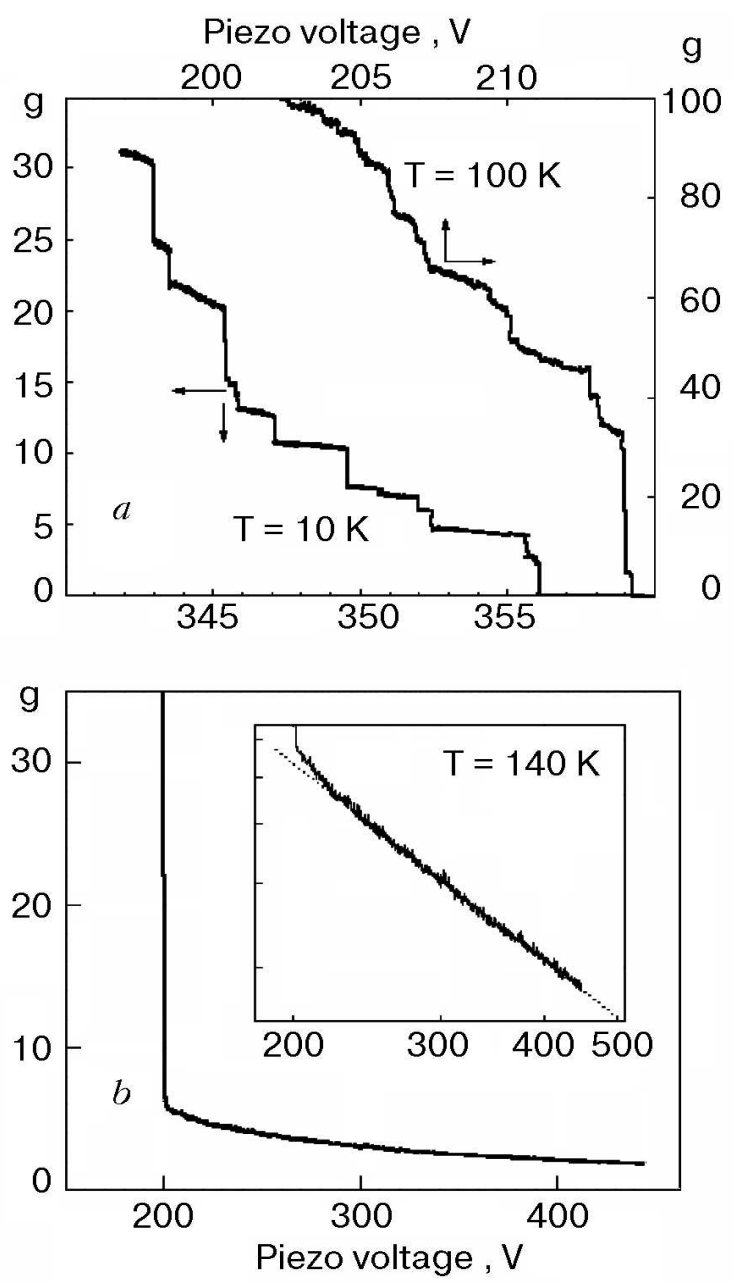

Fig. 3. $a-$ Typical individual scans for Li break-junctions at two different temperatures. 1 piezo-Volt corresponds approximately to $0.5 \mathrm{~A}$. $b$ - Long plateau at $140 \mathrm{~K}$, shown on a logarithmic scale in the inset.

means that putting the external force to zero the conductivity of the neck still evolves with time until the contact disappears.

We mentioned above that $\mathrm{Li}$ atoms revealed increased diffusion when we considered the low intensity of the $g=1$ peak in the Li histograms at helium temperatures. The enhanced mobility of the atoms also leads to the anomalous behavior of the conductance trace $G\left(V_{\text {piezo }}\right)$ at high temperatures shown in Fig. 3,b, where it steeply drops to zero from a fairly high conductance. For $\mathrm{Li}$ at $T=140 \mathrm{~K}$ all steps on the $G\left(V_{\text {piezo }}\right)$-scans disappear and all structure in the histograms disappears except at $g \approx 5$. This peak in the histogram becomes very high corresponding to anomalously long plateaus in the scans. The length of the plateaus reaches several tens of $\AA$, while typical plateaus correspond to less than $1 \AA$. The same qualitative behavior was observed for $\mathrm{Na}$, although for this metal it was more difficult to register scans at such elevated tempera- 
ture. We interpret this phenomenon in the following way.

At $T \approx 140 \mathrm{~K}$ the Li nanowire «melts», although the temperature of bulk melting point is much higher. By this we mean that the diffusion of atoms over the length of the nanowire becomes much faster than our data acquisition rate. According to Refs. 24, 25 a strong depression of the melting point is expected for small systems due to the Laplace tension. The change of the melting temperature $T_{m}$ compared to the bulk value $T_{0}$ is approximately given by $\left(T_{m}-T_{0}\right) / T_{0}=$ $=2\left(\sigma_{1}-\sigma_{2}\right) / Q R$, where $\sigma_{1,2}$ is the surface tension in liquid and solid phases, respectively, $Q$ is the heat of fusion, and $R$ is the wire radius [26]. Thus, we suggest that at the starting point of the long plateau a liquid-like metallic neck appears, which connects the solid banks. Further elongation of the neck proceeds continuously, decreasing its diameter as the wire is stretched, approximately as a power law $g \propto\left(V_{\text {piezo }}\right)^{n}$, with $n \simeq-1.3$.

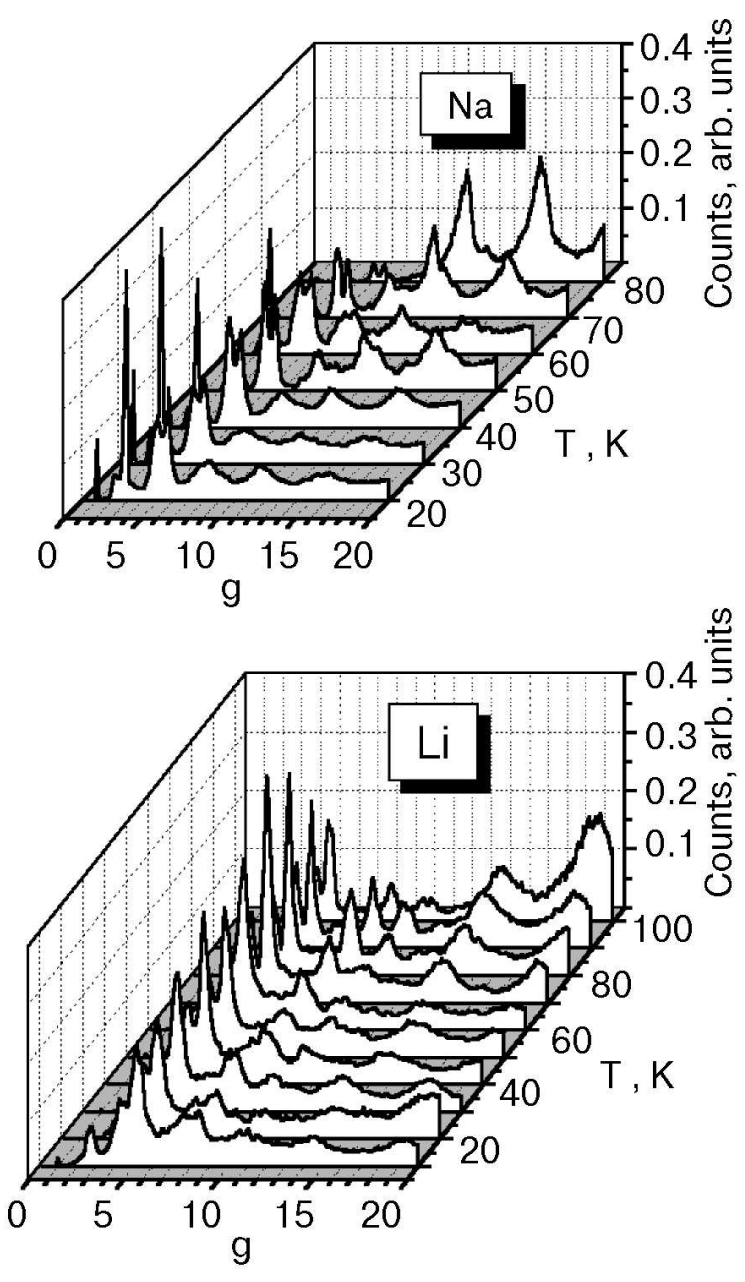

Fig. 4. Temperature evolution of conductance histograms for $\mathrm{Na}$ and $\mathrm{Li}$. The histograms have been normalized to the total area under the curves.
Temperature dependence of the histograms. The temperature dependences of $\mathrm{Na}$ and $\mathrm{Li}$ histograms are shown in Fig. 4. One recognizes the low temperature curves similar to those shown in Fig. 2. While raising the temperature, the low-conductance peaks gradually disappear due to increasing thermal instabilities. On the other hand, for thicker wires a new succession of strong peaks grows, owing to the increased mobility of atoms at elevated temperatures. We shall consider these series of peaks in detail in the next Sections. Here, we only point out that the positions of these peaks on the conductance axis does not correspond to those that might arise due to individual quantum modes, which would increment either by one or two conductance quanta. Instead, these peaks are broader and reveal a separation that grows with conductance. The intensities of these peaks depend, among other parameters, on the depth of indentation, which can approximately be characterized by the highest value of conductance scale in the histogram. A second parameter is the voltage bias, at which the scans are recorded. Unfortunately, there are additional parameters that we can hardly control with the technique used. These include possible surface contaminations and the orientation of the crystallites on the two electrodes. We stress that these drawbacks mainly concern the intensity of the peaks and much less their positions on the conductance axis, while the period of the peak series remains almost unaltered. Since, as shown below, there are two mechanisms leading to different series of peaks with different periods (namely, electronic and atomic shell series), the positions of the peaks depend upon the relative intensity of these mechanisms. Fortunately, the true frequencies of the oscillations can be obtained from the Fourier transform of the experimental histograms.

\subsection{Electronic shell and supershell effects}

In this Section we describe the observation of electronic shell-effect oscillations [11,12] in alkali metal nanowires. Experimental results depend on several factors discussed above. We emphasize once again that all shell-effect structures are observed at elevated temperature, at which atoms have enough mobility to occupy the most favorable positions in the lattice structure. This structure corresponds to the local minima of the total free energy of the nanowire.

Histograms and peak positions. A typical histogram for sodium is shown in Fig. 5, $a$. The positions of the main peaks, labeled by sequential numbers, are regularly spaced as a function of $g^{1 / 2}$. That 


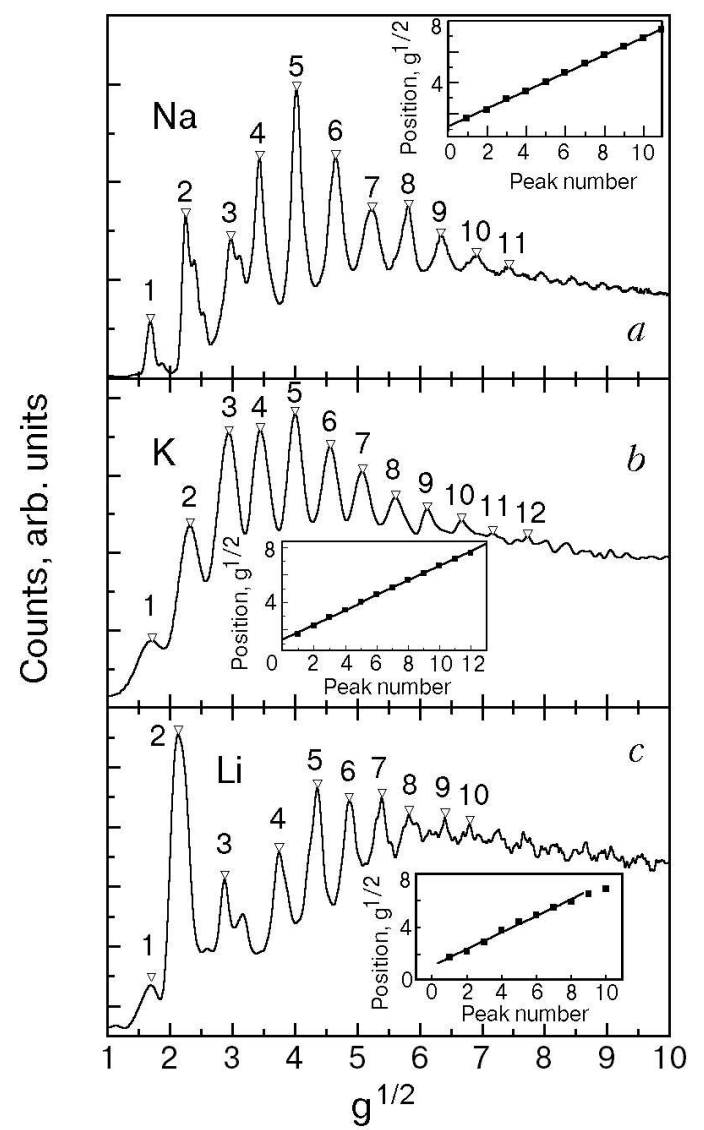

Fig. 5. Conductance histogram for sodium constructed from $\approx 14000$ individual traces recorded at $T=90 \mathrm{~K}$ and at $100 \mathrm{mV}$ voltage bias $(a)$; conductance histogram for potassium constructed from $\sim 5000$ individual traces recorded at $T=100 \mathrm{~K}$ and $8 \mathrm{mV}$ voltage bias $(b)$; conductance histogram for lithium constructed from $\sim 8000$ individual traces recorded at $T=100 \mathrm{~K}$ and $10 \mathrm{mV}$ voltage bias $(c)$. We count the split peak $\# 3$ as a single peak, as for $\mathrm{Na}$ (see $a$ ). The peaks are numbered as shown, and a plot of peak positions vs. their index number is fit by a straight line in the insets. The slopes are $0.581 \pm 0.005$, $0.54 \pm 0.005$, and $0.62 \pm 0.02$ for $\mathrm{Na}, \mathrm{K}$, and $\mathrm{Li}$, respectively. Note that the $x$-axis is given as the square root of $g$.

means that they periodically appear as a function of the radius of the wire, since for a circular nanowire the radius of the smallest cross section $R$, obtained from the Weyl semi-classical [27] expression for a ballistic regime, reads:

$$
g_{W} \approx\left(\frac{k_{F} R}{2}\right)^{2}\left(1-\frac{2}{k_{F} R}\right) \approx\left(\frac{k_{F} R}{2}\right)^{2},
$$

where $k_{F}$ is the Fermi wavevector and $\left(k_{F} R / 2\right)^{2}=g_{S}$ is the Sharvin conductance. We prefer to plot $g^{1 / 2}$ along the abscissa, since it is the experimentally measured quantity, while the inferred radius depends somewhat on the shape of nanowire. For example, for a contact in the form of an orifice $g_{W} \simeq g_{S}\left[1-1 /\left(k_{F} R\right)\right]$, which makes the correction to the Sharvin conductance smaller [16]. The inset in Fig. 5, $a$ demonstrates that the peak positions are periodic, with a period of $\sim 0.58$. We see that at elevated temperatures the $g=1$ peak is absent and the highest intensity is reached at $g \approx 16$. The shape of the background is determined by the global variation of the conductance with elongation, which in turn depends on the effective length of the nanowire that deforms during elongation $[21,28]$ (see below). This length is located near the narrowest cross section of the nanowire and can be qualitatively described with the model considered in Ref. 21. The latter is valid when diffusion of the atoms from the neck to the electrodes can be neglected ${ }^{*}$. Superimposed in the main periodic structure we note an oscillating behavior of the envelope of the maxima in the histogram of Fig. 5, $a$. This modulation is the signature of the supershell effect and will be considered in more detail in the section on the Fourier transformation. Finally, we draw attention to the small-intensity oscillations at $g^{1 / 2} \gtrsim 7.5$. They have a much smaller period and are due to the crossover from electronic to atomic shell effect (see below).

Similar periodic structure is seen for potassium (Fig. 5,b) and lithium (Fig. 5,c). The periods shown in the insets are 0.54 and 0.62 for $\mathrm{K}$ and $\mathrm{Li}$, respectively. Maximal amplitudes are observed at $g^{1 / 2} \simeq 4-5$, and the peak at $g=1$ is missing just as for $\mathrm{Na}$. The periods are close to what is observed in $\mathrm{Na}$, although a small change in the slope is often observed for different samples. The modulation of the peak heights also varies from contact to contact. While a modulation is clearly seen in the Li histogram (Fig. 5,c), for the given $\mathrm{K}$ contact (Fig. 5,b) only a non-monotonous behavior around the peaks with indices $3-5$ is found. In the next Section we present a different $\mathrm{K}$ histogram, which exhibits much more modulation by the superposition of different shell effects (Fig. 7).

Fourier transformations. Determination of the average period from the slope of the peak positions in terms of $g^{1 / 2}$ versus their indices (see insets in Fig. 5) gives limited information about the probabilities for observing specific conductance values. For example, in this way, one cannot account for the non-monotonous behavior of the peak heights.

* We shall discuss the transformation length [21] in the paragraph concerning the atomic shell structure. 

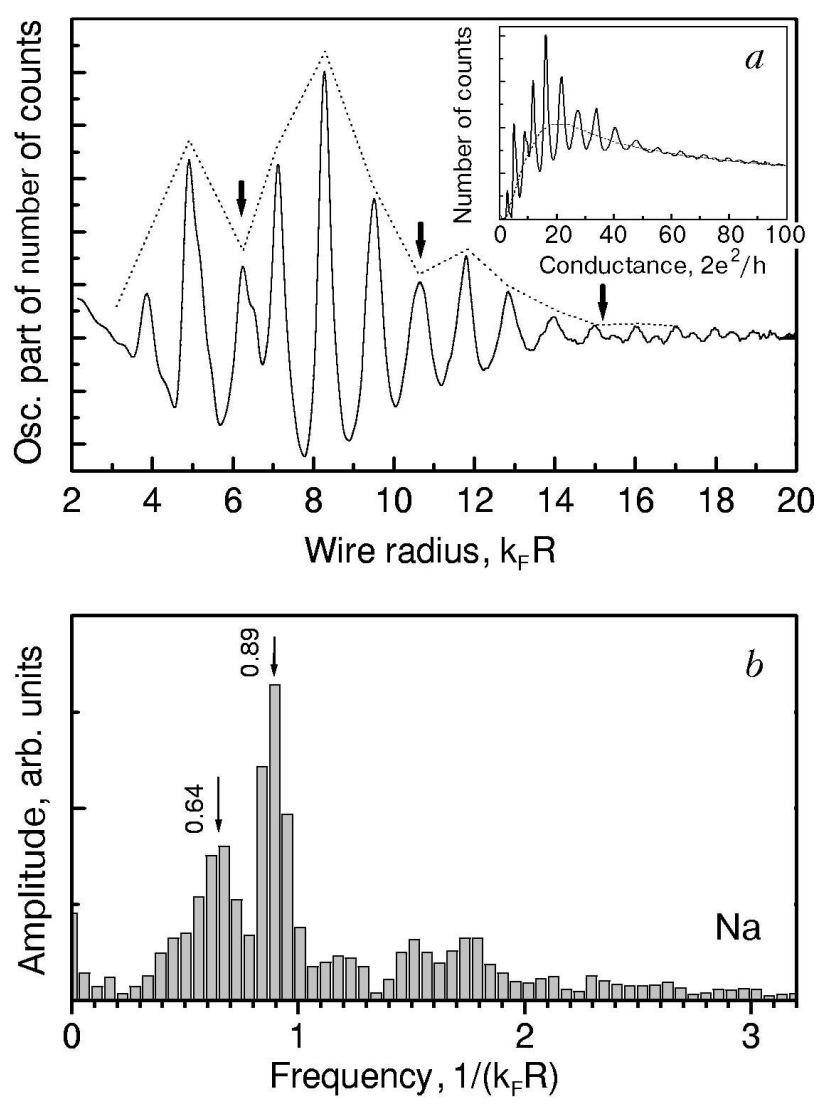

Fig. 6. $a-$ Conductance histogram for sodium from Fig. 5, $a$ with a smooth background subtracted (the original curve and the background are shown in the inset). The arrows point at the positions of the minima in the oscillation amplitudes. $b-$ Fourier spectrum of the curve in $a$.

The problem arises, whether to count the peaks with smaller amplitude.

Fortunately, we can make use of a direct Fourier analysis of the histograms, which exposes a superposition of several periodic components. A typical example for sodium is shown in Fig. 6. In order to avoid a huge low-frequency tail in the Fourier spectra, we subtract a smooth background shown by the dashed curve in the inset of Fig. 6,a. The resulting curve is displayed on the main panel of Fig. 6,a. It reveals a clear low-frequency modulation with a period of $\sim 4.0$. Here, we use the variable $k_{F} R$ instead of $g^{1 / 2}$ for the abscissa, by use of the Weyl expression (1), which will prove convenient for comparison with the theoretical results in the discussion section below. The Fourier spectrum for this histogram is shown in Fig. 6,b. It contains two prominent frequencies 0.64 and 0.89 , where the first has a smaller intensity than the second. The latter of the two frequencies is very close to the (average) one obtained for the magic numbers in spherical clusters [11]. As will be discussed below, for clusters the intensity of the first principal frequency is negligibly small, and the observed frequency equals the average of triangular and square orbits: $(0.83+0.90) / 2=0.86$ (see Table). The presence of the lower frequency component for the nanowires, explains why the frequency of the second prominent peak is a little bit larger than what would be obtained from the averaged period $\Delta\left(g^{1 / 2}\right) \simeq 0.58$ given by the slope in the inset of Fig. $5, a^{*}$. In Fig. $6, b$ the low intensity peaks at frequencies above 1 are presumably due to the

Table

First few shortest classical periodic orbits and their frequencies in circular geometry. The circle $(t=1, p=\infty)$ marks the end of the first band $(t=1)$; the second one contains trajectories which make two revolutions around the center $(t=2)$, the third three, etc.

\begin{tabular}{|c|c|c|c|c|}
\hline Shape & $t$ & $p$ & Perimeter $L$ & $\begin{array}{l}\text { Frequency } \\
f=1 / \Delta(k R)\end{array}$ \\
\hline & 1 & 2 & $4 R$ & $2 / \pi \approx 0.64$ \\
\hline & 1 & 3 & $3 \sqrt{3} R$ & $3 \sqrt{3} / 2 \pi \approx 0.83$ \\
\hline & 1 & 4 & $4 \sqrt{2} R$ & $2 \sqrt{2} / \pi \approx 0.90$ \\
\hline & $\cdots$ & $\cdots$ & $\cdots$ & $\cdots$ \\
\hline & 1 & $\infty$ & $2 \pi R$ & 1 \\
\hline & 2 & 4 & $8 R$ & $4 / \pi \approx 1.27$ \\
\hline & 2 & 5 & $10 R \sin \left(\frac{2 \pi}{5}\right)$ & $5 \sin \left(\frac{2 \pi}{5}\right) \approx 1.51$ \\
\hline & 2 & 6 & $6 \sqrt{3} R$ & $3 \sqrt{3} / \pi \approx 1.65$ \\
\hline
\end{tabular}

* The latter corresponds to the frequency 0.866 . 

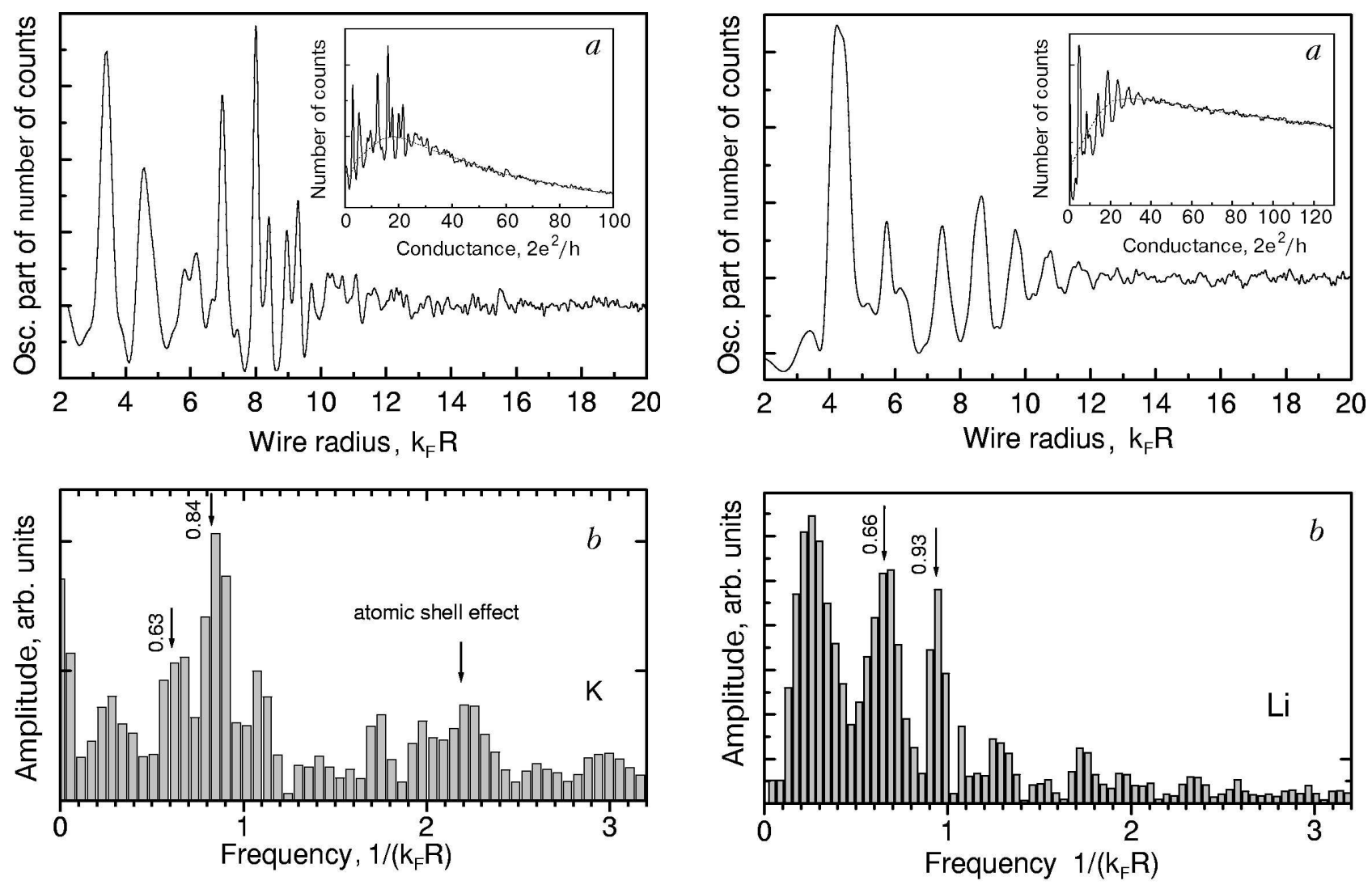

Fig. 7. $a-$ Conductance histogram for potassium at $T=50 \mathrm{~K}$, constructed from 1000 individual scans recorded at $125 \mathrm{mV}$ voltage bias, after subtracting a smooth background (the original curve and the background are shown in the inset). $b-$ Fourier spectrum of the curve in $a$.

harmonics and combinations of the two principal frequency.

Considering the histogram for potassium (Fig. $7, a$ ), which is different from the one in Fig. $5, b$, we see that there are again two prominent peaks in the Fourier transform, at 0.63 and 0.84 , close to those observed for sodium. In addition, there is an appreciable maximum around 2.2 , which is difficult to explain in terms of overtones, but stems from the atomic shell effect, as we will see below. For the given $\mathrm{K}$ histogram one obtains an average period of $\Delta\left(k_{F} R\right) \simeq 1.15$ from the slope of the peak positions, giving an average frequency of $1 / \Delta\left(k_{F} R\right)=0.87$. This nearly coincides with the upper principal frequency because of the small intensity of the first principal frequency.

Finally, let us consider the Fourier transform of the Li histogram from Fig. 5,c. Figure $8, b$ shows three peaks of about the same intensity. Those at frequencies 0.66 and 0.93 are of the same origin as the principal frequencies in $\mathrm{Na}$ and $\mathrm{K}$. As to the lowest frequency at 0.25 , it may be due to an anomalously strong interference of the principal

Fig. 8. $a$ - Conductance histogram for lithium from Fig. 5,c with a smooth background subtracted (the original curve and the background are shown in the inset). $b-$ Fourier spectrum of the curve in $a$.

frequencies, giving rise to a difference-frequency with a period of 4.2. This may be due to the fact that the intensity of the first principal peak (at 0.66 ) is as strong as the second one. It is interesting to note, that both principal Fourier frequencies in $\mathrm{Li}$ are higher than in $\mathrm{Na}$ and $\mathrm{K}$. However, the average frequency of $(0.66+0.93) / 2=0.78$ is lower than in $\mathrm{Na}$, corresponding to a larger average period $\Delta\left(g^{1 / 2}\right)=0.64$ (or $\left.\Delta\left(k_{F} R\right) \simeq 1.27\right)$. Indeed, since in $\mathrm{Li}$ the intensities of the two principal peaks are approximately the same, we can simply average their frequencies, while this is would not be correct for $\mathrm{Na}$ and $\mathrm{K}$.

\subsection{Atomic shell effect [13]}

Searching for the shell oscillations at still higher conductance, we have found a new series that appears to be due to the pure classical effect similar to that found in clusters. As in clusters, we named it an «atomic shell effect» and discuss its origin in the next Section. We start by presenting the main experimental facts concerning this effect. 


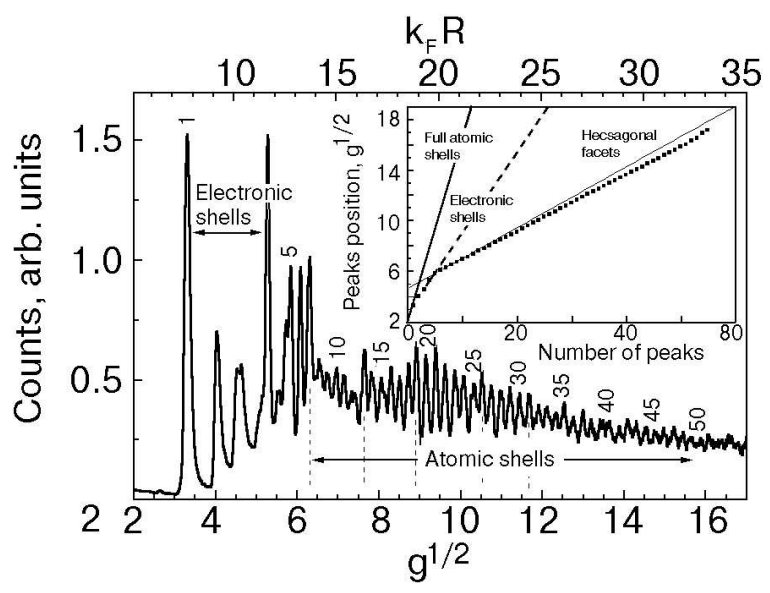

Fig. 9. Potassium histogram constructed from 1000 individual conductance traces recorded at $1 \mathrm{mV}$ voltage bias and at $T=100 \mathrm{~K}$. Peaks are sequentially numbered and their position is plotted as a function of this number in the inset. Straight solid lines show the slopes 1.447 and 0.241 for full atomic shells and single hexagonal facets, respectively. A linear fit for the electronic-shell points gives 0.61 (dashed straight line). A linear fit to the experimental data starting with number 6 gives a slope of 0.224 . Along with $g^{1 / 2}$ values on the $x$-axis the corresponding $k_{F} R$ values are given on top, according to formula (1).

Crossover from electronic to atomic shell effect. The most spectacular results are achieved for potassium. The corresponding histogram is shown in Fig. 9. It shows two types of oscillations with a relatively sharp crossover at $g^{1 / 2} \approx 6$. In the inset, the reduced conductances, corresponding to the peaks in the histogram, are plotted against their sequentially numbered indices. The index number of the peaks is also shown in the histogram, where they are labeled at increments of five. The first few points in the inset, corresponding to the strong peaks in the histogram, have an approximate slope of 0.61 , which agrees reasonably well with the slope determined for the electronic shells. Beyond the first few points, up to 48 points obey a linear relation with a slope of $0.225 \pm 0.001$. Structure in the histograms with this particular period has been reproducibly observed for many contacts. One can see that the transition between these two sets of oscillations (electronic and atomic) is quite sharp, although the shorter period pattern extends a little with reduced amplitude into the lower conductance range, where it overlaps with the electronic shell oscillations.

For sodium, the crossover is found at larger diameters than for potassium (Fig. 10) with periods equal to $(0.62 \pm 0.05) g^{1 / 2} \quad$ and $(0.224 \pm 0.001) g^{1 / 2}$ for electronic and atomic shells, respectively. For comparison in Fig. 10 we

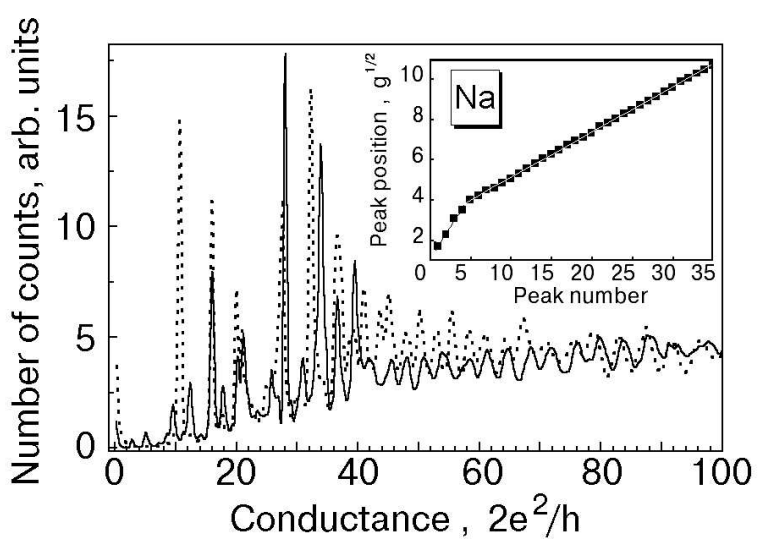

Fig. 10. Sodium histogram constructed from 10424 individual conductance scans recorded at $100 \mathrm{mV}$ voltage bias and at $T=100 \mathrm{~K}$. Peaks are sequentially numbered and their position vs. number is plotted in the inset. Straight-line fits give slopes of 0.62 and 0.224 for electronic and atomic series, respectively. One of the typical histograms for potassium is shown for comparison (dotted curve).

plot the histogram for the potassium nanowires as a thinner curve. Some discrepancies between the $\mathrm{Na}$ and $\mathrm{K}$ histograms are seen in the low conductance range, which are mostly influenced by the different voltage bias. However, the behavior remains basically the same.

Superposition of electronic and atomic-shell structure at low conductances. Atomic-shell oscillations can be observed at conductances starting as low as $g \approx 6-7$. In Fig. 11 we show some of the histograms recorded in a single series under seemingly the same experimental conditions. In the first recording no distinct oscillations were observed (not shown). Each trial, which consisted of many scans, was performed by making a contact with a resistance of about $1 \Omega\left(\sim 10^{4} \mathrm{~g}\right)$ and then mechanically receding the electrodes to bring the conductance down to the tunneling regime. The next several trials are displayed in Fig. 11 (curves 1-5). It is seen that for this particular sample the histograms change drastically from those resembling the atomic-shell oscillations (curves 1,2 and 3) to ones having the electronic shell periodicity (curves 4 and 5). Presumably, this is due to the increase in the mobility of the surface atoms. Namely, for the last trials the mobility of atoms at the surface is increase so much that no crystalline facets (see below) could be created during acquisition time for the scans. Other causes may be the random crystal orientation of the neck, creating a favorable condition for one of the two shell effects, and altering the shape of the nanowire during many indentations. Interestingly, the extreme shape of histograms presented in 


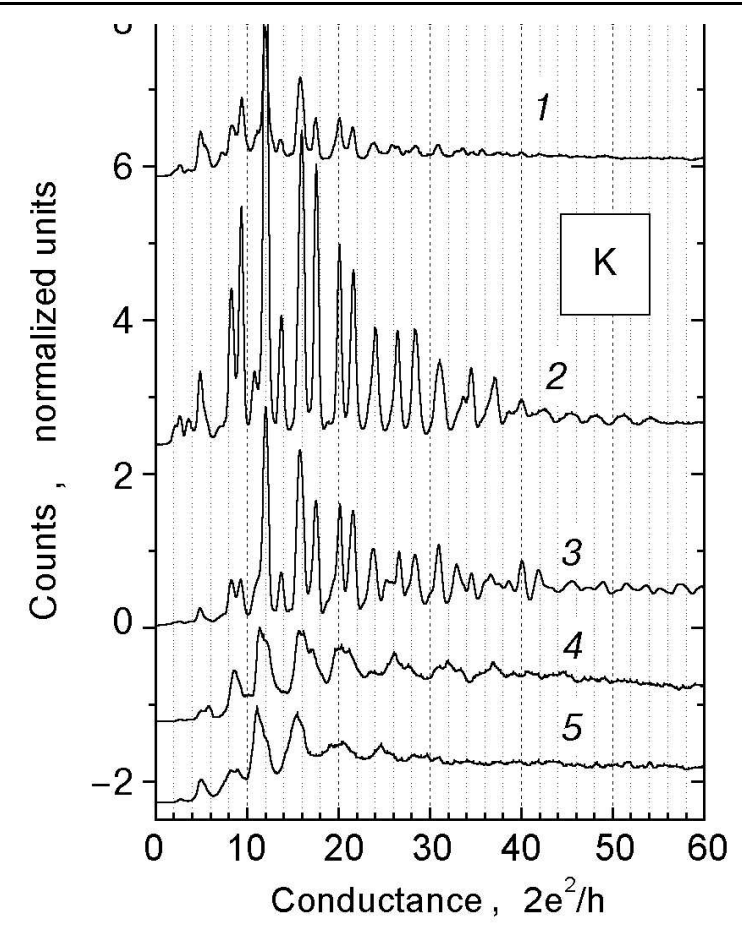

Fig. 11. Evolution of shell-oscillations obtained in successive runs in the same experiment for a potassium nanowire at $T=80 \mathrm{~K}, V_{\text {bias }}=10 \mathrm{mV}$. In curves 2 and 3 the atomic-like period is clearly seen, while for curves 4 and 5 the period is due to the electronic shell effect. Each histogram consists of several thousands up to several tens of thousand scans.

Fig. 11 (curves 4 and 5) reproduces quite satisfactorily the electronic-shell histograms presented above. The same is true for the atomic-shell histograms at low conductances (curves 2 and 3 ). As an illustration, in Fig. 12, $a$ we show by a thick line the histogram for another sample (recorded in an experiment approximately a year later) and superimposed on curve 2 from Fig. 11 (note the change in the $x$-coordinate). The reproducibility of the peak positions is quite good, despite important variations in the amplitudes. One should not think that the electronic-shell structure is negligibly small for these curves. The Fourier transformation shown in Fig. 12,b for the full curve in Fig. 12,a clearly shows the characteristic frequencies for both shell effects: electronic peaks at 1.2 and 1.8 and the atomic peak at 4.4. Other features with lower intensity may be explained as harmonics and subharmonics of the principal frequencies.

Additional structure of atomic-shell oscillations. Occasionally, the atomic-shell oscillations reveal additional structure in their amplitude and periodicity. The peaks can be grouped by sixes, based on the amplitudes, as seen for example in Fig. 9. Here, the thin vertical lines at $g^{1 / 2} \approx 6.3,7.6,8.9$, and 10.5 indicate higher amplitudes and correspond
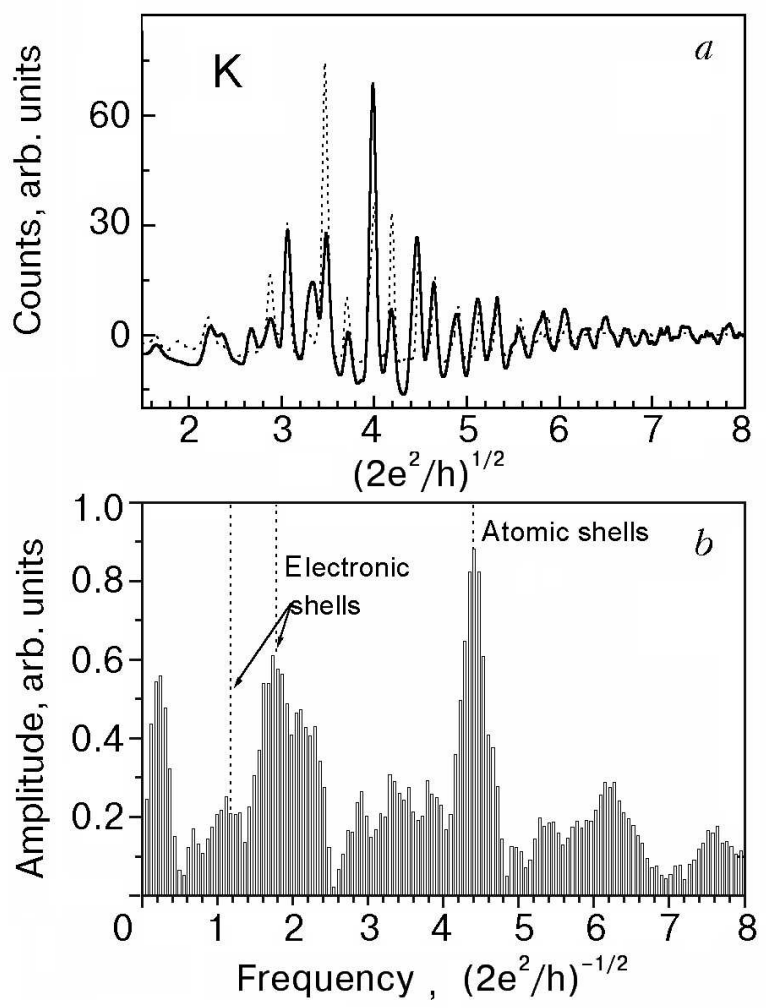

Fig. 12. $a-$ Two conductance histograms for potassium showing mainly atomic-shell oscillations. The dotted curve is curve 2 from Fig. 11, while the full curve was taken almost a year later. Good reproducibility is seen for the measurements. $b-$ Fourier spectrum of the full curve in $a$. Superposition of two shell effects is seen from the peaks in the Fourier spectrum at 1.2 and 1.8 , and 4.4 for electronic and atomic principal frequencies, respectively. $T=80 \mathrm{~K}, V_{\text {bias }}=10$ and $20 \mathrm{mV}$ for the dotted and full curves, respectively.

to the index numbers $7,13,19$, and 26 . For other contacts, there may be other envelope patterns. The period of these higher amplitude peaks equals $1.36 \approx 0.224 \times 6$, which is very close to a single close-packed monatomic layer, covering the nanowire (see below). Moreover, the period between adjacent peaks as a function of the reduced conductance $g$ appears not to scatter randomly around a linear dependence as one might expect. A linear dependence follows when the period is constant as a function of $g^{1 / 2}$. Instead, when the distance between the adjacent peaks is averaged over 3 points (as shown in the inset of Fig. 13) in order to smoothen fluctuations, they can again be grouped by approximately six peaks (Fig. 13). For this particular contact, the averaged period exhibits a smooth staircase where the vertical steps closely coincide with the higher amplitude peak indices (numbers 7, 13, 19, and 26). The difference in 


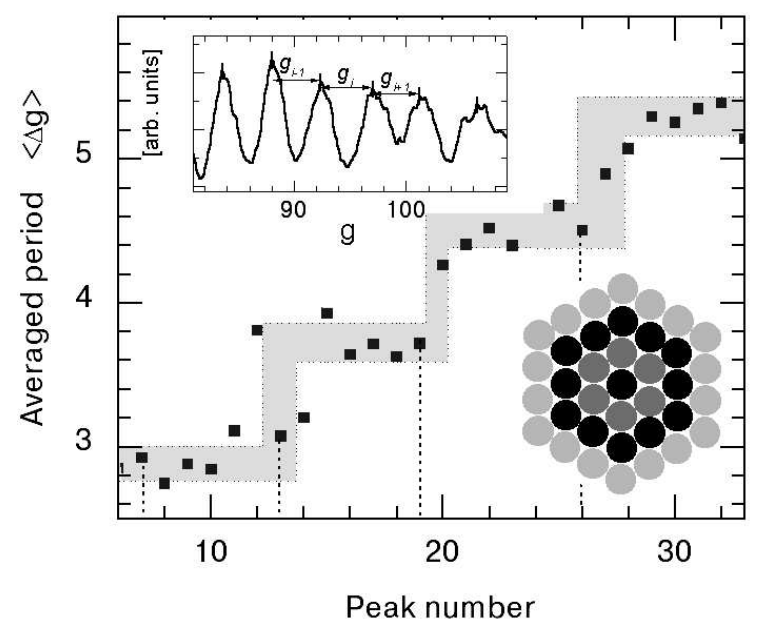

Fig. 13. The averaged period of the oscillations in Fig. 9 plotted vs. peak index. Vertical dotted lines mark the positions of peaks with maximal amplitude (numbers $7,13,19,26$ ). The gray staircase is a guide to the eye to identify the step structure. Part of a conductance histogram is shown in the inset to explain the averaging procedure $\left\langle\Delta g_{i}\right\rangle=1 / 2\left[\left(g_{i}-g_{i-1}\right)+\right.$ $\left.+\left(g_{i+1}-g_{i}\right)\right]$. Artist impression of atomic positions in the normal cross section of the wire is sketched in the lower right corner. For simplicity, we draw the two-dimensional structure. Six facets symbolize six data points at each plateau, while the height of the step corresponds to successive layers for a close packed structure.

height of the plateaus is approximately one $G_{0}$. This behavior will be explained in the next Section.

\section{Discussion}

\subsection{Electronic shells}

Exact quantum mechanical result. To understand semi-quantitatively the electronic shell effect in the formation of metallic nanowires, we use the simplest model of a metallic circular cylinder $[15,29,30,33]$, which connects two metallic reservoirs (electrodes). A more realistic shape for the wires [21] will be considered later. When the wire diameter $d$ is comparable with the De Broglie wave length of conduction electrons at the Fermi energy, quantum size effects in the lateral dimensions lead to a finite number of conductance channels, each corresponding to an electron wave propagating along the wire axis $z$. In order to obtain the energy spectrum of these modes $\varepsilon\left(k_{z}\right)$, one solves the Schrödinger equation to obtain

$$
\varepsilon\left(k_{z}\right)=\varepsilon_{m n}+\frac{\hbar^{2} k_{z}^{2}}{2 m_{e}}
$$

where

$$
\varepsilon_{m n}=\frac{\hbar^{2}}{2 m_{e} R^{2}} \gamma_{m n}^{2}
$$

$m_{e}$ is the electron mass, $\gamma_{m n}$ is the $n$th zero of the $m$ th Bessel function of the first kind, $R$ is the radius of the nanowire, $d=2 R$, and $k_{z}$ is the $z$-component of the electron wave number. In any single one-dimensional sub-band the total number of states per unit length of the wire (including spin degeneracy) is

$$
N_{m n}(\varepsilon)=2 \frac{2\left|k_{z}(\varepsilon)\right|}{2 \pi}=2\left(\frac{2 m_{e}}{\pi^{2} \hbar^{2}}\right)^{1 / 2} \sqrt{\varepsilon-\varepsilon_{m n}},
$$

and the density of states is

$$
D_{m n}\left(\varepsilon-\varepsilon_{m n}\right) \equiv \frac{d N_{m n}(\varepsilon)}{d \varepsilon}\left(\frac{2 m_{e}}{\pi^{2} \hbar^{2}}\right)^{1 / 2} \frac{1}{\sqrt{\varepsilon-\varepsilon_{m n}}} .
$$

Let us now consider a cylinder of finite length $L$, radius $R$ and volume $V$ being elongated at the ends so that its volume $V=\pi R^{2} L$ remains constant. To use expressions (2) $-(4)$ we should impose the condition $L>R$ which allows us to consider the cylinder as quasi-infinite in the $z$-direction. The total number of the occupied states in such a cylinder filled up to the bulk Fermi energy $\varepsilon_{F}$ is ${ }^{*}$

$$
\begin{gathered}
N\left(\varepsilon_{F}\right)=\sum_{m, n} N_{m n}\left(\varepsilon_{F}\right)=\sum_{m, n} \int_{\varepsilon_{m n}}^{\varepsilon_{F}} L D_{m n}\left(\varepsilon-\varepsilon_{m n}\right) d \varepsilon= \\
=\sum_{m, n} 2 L\left(\frac{2 m_{e}}{\pi^{2} \hbar^{2}}\right)^{1 / 2} \sqrt{\varepsilon-\varepsilon_{m n}} .
\end{gathered}
$$

The total energy of the electron system is

* The assumption that the Fermi energy of the wire is always equal to that of the bulk is, generally speaking, invalid [31]. This correction, however, does not change the results qualitatively and, since we are only interested in the periodicities of the thermodynamic quantities, it can be ignored for the sake of simplicity. 


$$
\begin{gathered}
E=\sum_{m, n} \int_{\varepsilon_{m n}}^{\varepsilon_{F}} L \varepsilon D\left(\varepsilon-\varepsilon_{m n}\right) d \varepsilon= \\
=\sum_{m, n}\left[\frac{2}{3} L\left(\frac{2 m_{e}}{\pi^{2} \hbar^{2}}\right)^{1 / 2}\left(\varepsilon_{F}-\varepsilon_{m n}\right)^{3 / 2}+\varepsilon_{m n} N_{m n}\left(\varepsilon_{F}\right)\right] .
\end{gathered}
$$

Assuming $\mu=\varepsilon_{F}$ at low temperatures we can write the thermodynamical potential

$$
\Omega=E-\mu N=-\frac{4}{3} L\left(\frac{2 m_{e}}{\pi^{2} \hbar^{2}}\right)^{1 / 2} \sum_{m, n}\left(\varepsilon_{F}-\varepsilon_{m n}\right)^{3 / 2},
$$

whose derivative with respect to the length gives us the force
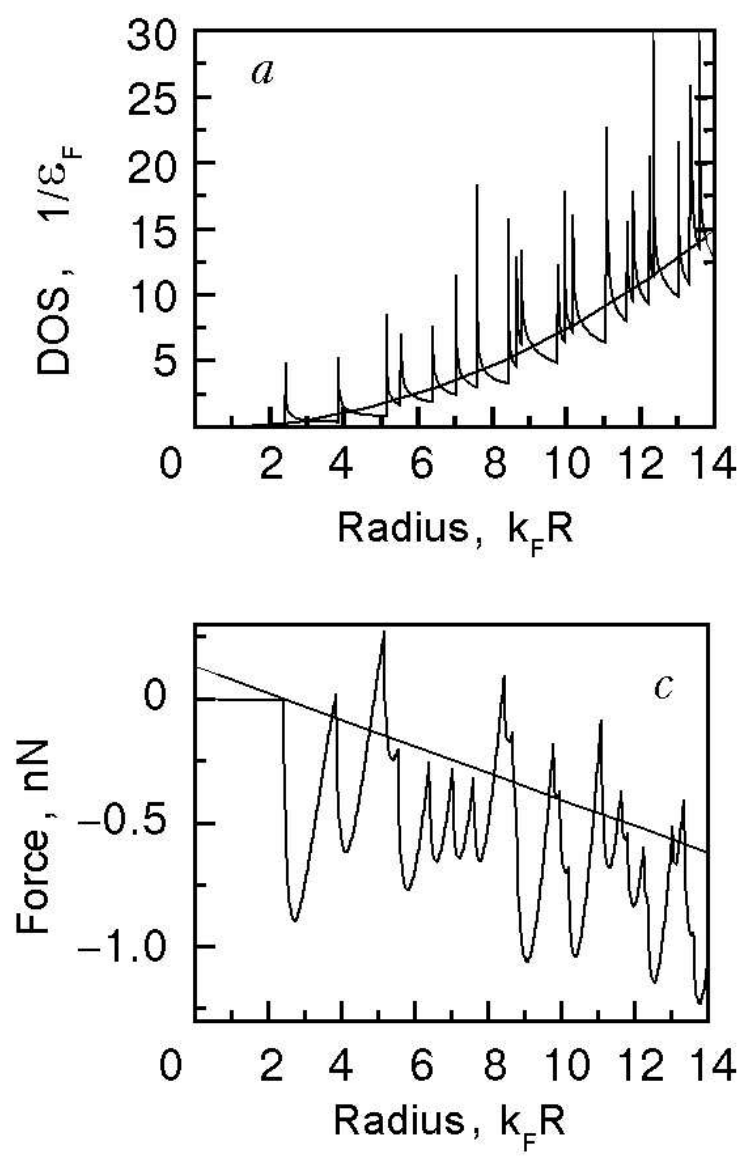

$$
\begin{gathered}
F=-\frac{d \Omega}{d L}= \\
=\left(\frac{2 m_{e}}{\pi^{2} \hbar^{2}}\right)^{1 / 2} \sum_{m, n}\left(\frac{4}{3}\left(\varepsilon_{F}-\varepsilon_{m n}\right)^{3 / 2}-2 \varepsilon_{m n}\left(\varepsilon_{F}-\varepsilon_{m n}\right)^{1 / 2}\right)
\end{gathered}
$$

The density of states, the thermodynamic potential and the force are calculated as a function of the wire radius for a given Fermi energy using the formulae
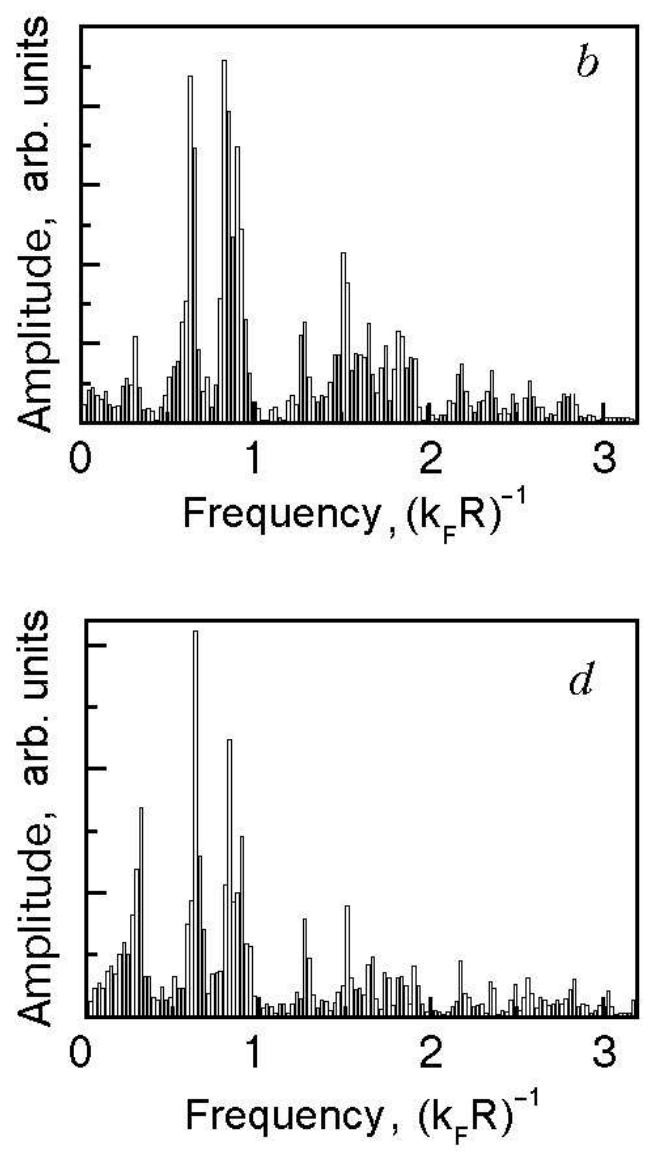

Fig. 14. Density of states (DOS) $a$ and force $c$ in the nanowire calculated using Eq. (5). Smooth backgrounds, subtracted before performing the Fourier transforms, are also shown. $b$ and $d$ show Fourier transforms of the oscillating parts of DOS and the force, respectively. 


$$
\begin{gathered}
D\left(k_{F} R\right)=\frac{1}{\pi \varepsilon_{F}} \sum_{m, n}\left[1-\left(\frac{\gamma_{m n}}{k_{F} R}\right)^{2}\right]^{-1 / 2}, \\
\Omega\left(k_{F} R\right)=-\frac{2 \hbar^{2} k_{F}^{5} V}{3 \pi^{2} m_{e}} \frac{1}{\left(k_{F} R\right)^{2}} \sum_{m, n}\left[1-\left(\frac{\gamma_{m n}}{k_{F} R}\right)^{2}\right]^{3 / 2} \\
\times \sum_{m, n}\left[\frac{2}{3}\left(1-\left(\frac{\gamma_{m n}}{k_{F} R}\right)^{2}\right)^{3 / 2}-\frac{\hbar^{2} k_{F}^{3}}{\pi m_{e}} \times\right. \\
\left(k_{F} R\right)^{2}
\end{gathered}
$$

The results for a sodium nanowire with a constant volume of $10 \mathrm{~nm}^{3}$ and the bulk Fermi energy are plotted in Fig. 14. Whenever the bottom of another sub-band falls below the Fermi energy, the density of states and the force reveal singularities. The force, being the derivative of the thermodynamic potential with respect to the length of the cylinder, describes the response of the system to elongation. When its value is non-zero the wire tends to shorten (negative force) or stretch (positive force) spontaneously in order to reduce its free energy; only when the force is zero a true state of stability is achieved. We should also take into account that in the classical limit of large diameters we should recover a constant destabilizing force resulting from the surface tension. Therefore, we do not have truly stable nanowires, but rather those with enhanced stability at the radii where the force is minimal. Those minima in the absolute value of the force give the diameters at which the wires are particularly stable. At the lowest conductances these minima occur synchronously with the conductance jumps, coinciding with the maxima in the density of states. But when multiple singularities of the spectrum are very close to each other forming a bunch (like ones at $k_{F} R \approx 8.5,10.5,12$ etc.) the force shows one global extremum. This bunching and the corresponding pronounced force extrema mark the fundamental differences between the con- ductance quantization and shell effects: the former stems from individual spectral singularities, while the latter is due to the inhomogeneous distribution of the features in the spectrum (caused by geometrical symmetries), which results in their periodic grouping, or bunching. At this point we cannot say anything about the periodicities of the bunching as a function of radius. While it is easy to calculate them numerically for not too large $k_{F} R$, an exact analytical approach using the double Poisson summation formula [32] is complicated and a much more transparent derivation based on the semi-classical Bohr-Sommerfeld quantization condition is used instead. Even if we plot the density of states (or any other quantity dependent on it) numerically on a large scale, the periodicities are not yet seen with a naked eye (Fig. 14,a). So naturally we would like to take a Fourier transform of exactly calculated thermodynamic quantities (5) to see whether the periodicities are indeed there, and we follow the same procedure as we used above for experimental data processing. Thus, we simply subtract the smoothed background by hand (see the smooth curves in Figs. 14, $a$ and $c$ ) and perform the Fourier transformation. Both frequency spectra (Figs. 14,b and $d$ ) give us three prominent peaks ${ }^{*}$ at $0.63,0.83$ and 0.90 . The latter two are very close to each other, and, taking into account the reduced amplitude of the third peak and its proximity to the second one, we can say that the oscillating part of the density of states as well as the force is mainly composed of two major frequencies, 0.62 and 0.87 . These correspond to the two peaks in the experimental Fourier spectra at 0.64 and 0.89 (Fig. 6). This is a very important result, which shows that quantum oscillations in each of the quantities of Eq. (5) possess the principal periodicities of the energy spectrum, and that this seemingly irregular spectrum of electron energies in a cylindrical nanowire can be decomposed into a few basic harmonics.

Semiclassical approximation. Much more information about bunching of energy levels, leading to periodicities in the spectrum, can be obtained from the semiclassical approach [30,34-36]. In this approach we consider an electron as a wave packet constructed from many quantum mechanical eigen-

* The features at about 1.3, 1.6 and higher are the higher-order harmonics of the main frequencies; their presence will be explained later. The minor peak at 0.3 is probably a sub-harmonic due to the size of the interval of transformation. This «extra» feature is an artifact of our crude method - it is absent if one treats the problem exactly [35]. 
states. The wave packet has wave vectors $k$ concentrated in the range satisfying the uncertainty principle in the space region, where we would like to localize our particle. For a circular cross section of the nanowire, the condition for validity of the semiclassical approximation reads $k R>>1$. Instead of finding the discrete quantum mechanical eigenvalues, one uses the approximate Bohr-Sommerfeld semi-classical quantization condition:

$$
\frac{1}{2 \pi \hbar} \oint p d x=v+\frac{1}{2}
$$

where $v$ is a positive integer, $p$ is the momentum and the integration is performed over one period of classical motion of the particle in the plane perpendicular to the wire axis.

For an axially symmetric circular cylinder, this condition transforms into a one-dimensional quantization condition along the radius variable, of the form [37]:

$$
\int_{m / k}^{R} \sqrt{k^{2}-m^{2} / r^{2}} d r=\pi(n+\varepsilon / 4),
$$

where $k=\sqrt{2 m_{e} E} / \hbar, E$ is the kinetic energy, $m=\ldots 2,-1,0,1,2, \ldots$ is the azimuthal quantum number, $n$ is a positive integer, $\varepsilon / 4<1$, and $r$ is the wire radius, which has two turning points: $m / k$ and $R$, respectively. Integrating Eq. (6), we obtain the quantization condition for $k$ :

$$
\begin{gathered}
k R(\sin \varphi-\varphi \cos \varphi)=(n+\varepsilon / 4) \pi, \\
\cos \varphi=m /(k R) .
\end{gathered}
$$

This is an implicit dependence of $k$ on $n$ and $m$ from which the semiclassical spectrum can be derived.

In the case of a complicated geometry, this approach is preferred to solving the Schrödinger equation exactly, but in our simple symmetric case we can obtain the spectrum exactly (see above) and therefore we shall rather use this relation to analyze the behavior of $k$. Following Balian and Bloch [38], we shall look for the bunching of the eigenvalues those intervals of $k$ where the energy levels are very close to each other. In order to find them, we should consider the variations $\delta m$ and $\delta n$ and find the conditions at which the corresponding $\delta(k R)<<1$ for some fixed $R$. As we are looking at the semiclassical approximation, we should consider only the part of the spectrum for large $k$ and $n$ where we can ignore $\varepsilon$. Combining both equations in (7) and varying $m$ and $n$ we obtain:

$$
\delta(k R) \sin \varphi=\pi \delta n+\varphi \delta m ; \quad \delta n, \delta m \in Z .
$$

In order to have $\delta(k R)=0$ for nonzero $\delta m$ and $\delta n$, the value of $\varphi$ should be a rational fraction of $\pi: \varphi / \pi=t / p$. Of course $\varphi$ has to satisfy Eqs. (7), which have solutions of the form [38] $\varphi=(t / p) \pi+O(1 / k R)$, where $p$ and $t$ are two integers, satisfying the condition $p \geq 2 t$. Since they enter only in a form of a fraction $t / p$, we can assume that they are co-prime.

We can now fix the values of $t, p \neq 0$ and look for the corresponding periodicity in $k R$. Substituting $\varphi=t \pi / p$ into the same variation (8), we obtain

$$
\Delta(k R) \sin \varphi=\frac{\pi}{p}(p \Delta n+t \Delta m) .
$$

In order to determine the period of this dependence, we have to find the smallest possible value of $\Delta(k R) \neq 0$. Following Bezout's theorem, for the integers $p$ and $t$ prime to each other there exist integers $\Delta n$ and $\Delta m$ such that $p \Delta n+t \Delta m=1$. Therefore, we obtain the periodicity of bunches: $\Delta k=2 \pi /(2 p R \sin \varphi)$. On the other hand, it is easy to see that $L(p, t)=2 p R \sin \varphi, \varphi=t \pi / p$, is just the perimeter of a polygon inscribed into a circle of a radius $R$ having $p$ vertices and making $t$ revolutions around the center. Therefore we see that our spectrum will contain multiple sets of periodical bunches of levels determined by the choice of $p$ and $t$ (or, alternatively, $\Delta n$ and $\Delta m$ ), and each set can be described as a closed semiclassical trajectory inside a circular cross section of a cylinder. Some of them are shown in Table. This is a very general result: it allows one to take a full set of eigenfunctions of the system determined by varying two quantum numbers $m$ and $n$, and using the above mentioned relations to «create» particles in the form of wave packets moving along certain trajectories (polygons inscribed in a circle).

Now let us compare the frequencies, which we obtain in exact quantum mechanical treatment (Fig. $14, b$ and $d$ ), with the ones, determined by semiclassical trajectories. As the number of vertices of a polygon with winding number $t=1$ inscribed in a circle grows, its perimeter approaches the circle itself. Thus, the minimum period $\Delta(k R)=2 \pi R / L(p, t)=1$ would be for a trajectory which is the circle itself, setting the maximum frequency in the spectrum. The positions of the first few periods are calculated and the corresponding trajectories are shown in Table.

It is important to note that the values that we obtain for the frequencies of the first few orbits coincide perfectly well with the positions of the 
peaks on the Fourier spectrum of the calculated electronic density of states (or force) in a perfect cylinder (Fig. 14,b and $d$ ). We can therefore make an important conclusion, namely, that the peaks in the Fourier spectrum of the oscillating part of the force in the nanowire, located at the frequencies of Table, each correspond to a single semiclassical trajectory. Every trajectory is characterized by two parameters $(t, p)$ and is composed of all eigenstates with quantum numbers $(m, n)$ chosen such that $\Delta m=p, \Delta n=t$. In other words, by grouping together the wave functions $\Psi_{m n}$ based on this principle $(\Delta m=p, \Delta n=t$, where $p$ and $t$ are fixed), one can construct a wave packet or a «particle» traveling along a classical trajectory of a polygon with $p$ vertices inscribed in a circle and making $t$ turns around the center.

There is an important difference between cluster physics and that of nanowires. In spherical clusters weight in the spectrum due to the diametric orbit $(t, p)=(1,2)$ is much smaller than that of triangular and square orbits". Then, in order to observe the beating between the latter two, one has to register a large number of oscillations, since the first minimum occurs at about 11-12 maxima due to the closeness of the frequencies. This is not a simple task because of the strongly decaying amplitudes, but one can make use of an additional feature, because the nearly identical intensities of triangular and square orbits give rise to the missing of one half of the oscillation period at the minimum [39]. In the nanowire, due to a less symmetric configuration, the intensity of the diametric orbit is approximately similar to that of the triangular and square ones (see Fig. 14). Thus, the beating between them is observed at much higher frequency, which can be easily seen in experimental histograms. The missing half-period is not observed in nanowire, because of the small number of oscillations between the nodes in the beating pattern.

The intensities of the spectral contributions of each trajectory are determined by their weight in the semiclassical expansion. From Figs. 14, $b, d$ one can see that for all $p>4$ and $t>1$ the contributions become negligibly small. This can be explained if one recalls that $p=\Delta m, t=\Delta n$, so the smaller these are, the more individual energy levels (or wave eigenfunctions) for a given limiting Fermi energy $\varepsilon_{F}$ will be present in the spectrum and contribute to a particular trajectory, increasing its weight in the spectrum. An exact derivation of this fact can be found in Ref. 38 .

\subsection{Atomic shells}

Structure of alkali nanowires: 6-fold symmetry. Recently several papers have appeared that consider unconventional structures for nanowires [40-42]. In Ref. 40 a 5-fold cluster-like structure was observed for a sodium nanowire through computer simulation. For gold, Kondo and Takayanagi claimed to observed spiral-like shell structures with an incremental number of 7 atoms in cross section [42]. Molecular dynamic simulations for the $s-p$ metals $\mathrm{Al}$ and $\mathrm{Pb}$ were reported in Ref. 41, where a «weird» spiral-like structure was predicted for nanowires with conductances smaller than $10 G_{0}$. Some of these weird wires resemble the structures observed in Ref. 42. Note that for thicker nanowire the calculations predict a close packed hexagonal structure.

When searching for an explanation for the structure in our experiment that we named «atomic» we are again led by the research on metal clusters. For alkali metal clusters produced in vacuum a clear transition has been observed between the series of magic numbers given by the closing of electronic shells, and the other series of magic numbers determined by the closing of geometric shells of atoms [8-10]. The latter series result from the fact that the clusters have a highly symmetric crystalline shape and the surface energy of a cluster attains a minimum when a new layer of atoms completely covers the surface. Let us assume that nanowires have a similar tendency to the crystalline order, and form facets. Such faceting has indeed been observed recently in transmission electron microscope images of gold nanobridges under thinning of the bridges by the electron beam current [43]. If many hundreds of wires are drawn, the facets should be expressed due to a well-defined minimum in the surface energy. Since the periodic pattern extends to large wire diameters, we first start with the assumption that the lattice structure in the wire is that of the bulk metal. The bulk lattice structure of potassium is bcc and the lowest energy surfaces are the [110] surfaces. A wire with only [110] facets can be formed with the wire axis along [100] and [111]. The cross section of the former would be

* Any polygon inscribed in a diametric cross-section of a sphere has an additional degree of freedom, being the rotation around an axis lying in the plane of the polygon. Only the diametric orbit does not have this feature, hence its contribution in the spectrum is reduced. 
square while that of the latter is a hexagon. The hexagonal wire clearly has a smaller surface area and would be preferred.

In order to calculate the slope of $g^{1 / 2}$ versus the peak index $i$, we approximate the relation between the conductance and wire cross section $A$ by the lowest order term, $g \approx k_{F}^{2} A / 4 \pi$, which is appropriate for $k_{F} R>>1$. The distance between the [110] atomic layers is $h=a_{0} \sqrt{2}$, where $a_{0}$ is the size of the cubic unit cell. We thus obtain,

$$
\frac{\partial \sqrt{g}}{\partial i}=\frac{3^{1 / 4}}{2 \sqrt{\pi}} k_{F} a_{0} .
$$

For a bcc lattice $k_{F} a_{0}=\left(6 \pi^{2}\right)^{1 / 3}$ and for the slope we obtain a value $\alpha=1.447$, which is shown in the inset in Fig. 9 as a line labeled «full atomic shell». This is clearly a much higher slope than the one we observe, and even higher than the slope for the electronic shell structure.

Sodium has a low-temperature martensitic phase transition toward a close packed atomic structure and it is possible that the surface tension favors close packing in nanowires. It turns out that one can construct a similar shape of a nanowire, as proposed above for the bcc structure having closed packing. Such a wire would have a fcc lattice, with the wire axis along [011] and six facets, that are

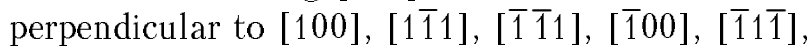
and $[11 \overline{1}]$. For this arrangement we obtain $\alpha=3^{5 / 6} \pi^{1 / 6} / 2^{13 / 12}=1.427$, which is very close to the value for the bcc structure.

When we assume that a stable configuration is obtained each time when a single facet of the nanowire is completed, in analogy to what is observed for some metal clusters (e.g., for aluminum $[10,44])$, then the slope becomes a factor of 6 lower, equal to 0.241 , in close agreement with the experimental data. Further support for this interpretation comes from considering the conductance values for closing of a full shell. We can write

$$
g^{1 / 2}=\alpha\left(j+j_{0}\right)
$$

where $j$ is the index of a full shell of atoms, with all facets covered, and $j_{0}=0.5$ is an offset value, which depends somewhat on the boundary conditions for the electrons. From this expression, and using the experimental value $\alpha=6 \times 0.224=1.34$, we find that the beginning of the atomic-shell structure in Fig. 9 is described by the shell numbers $j=4,5$, and 6 , which are closed at $g^{1 / 2}=6.02,7.36$, and 8.70 , respectively. Apart from the small shift, which may be absorbed in a new choice of the offset $j_{0}=0.66$, these values correspond to the higher intensity peaks with indices 7,13 and 19, and five smaller peaks between them. By analogy to the observation on Ca clusters [10], this suggests an enhanced stability for nanowires with all six facets covered. Alternatively, one can check the self-consistency of the linear relation (9) by assuming that the first fully covered layer with 6-fold symmetry should contain at least 6 alkali atoms in the cross section, taking into account that 1 monovalent atom approximately corresponds to a single conductance channel and, correspondingly, the reduced conductance $g \approx 6$. Thus, following formula (9), for the slope $\alpha$ we obtain $\sqrt{6} /(1+0.66)=1.47$, very close to the theoretical value 1.447 and the experimental slope 1.34.

Furthermore, proceeding this way, we obtain the explanation of the step structure shown in Fig. 13. When we remove subsequently 6 hexagonal facets with the same number (on the average) of atoms (see the inset in Fig. 13), they should give 6 successive stability peaks, which increase the conductance $g$ by the same average increment, depending on the size of the hexagon. While receding the electrodes, the next full atomic shell should give us the next 6 stability facets with an increment decreasing approximately by one atom, that is by one conductance quantum, etc. This is just what is observed in Fig. 13, where the edges of «plateaus» coincide with the maximal peaks with indices 7, 13, 19, and the increment between successive «plateaus» amounts to about 1 quantum unit.

One-atomic-layer slab model for stretching nanowire. In this Section we show that the hexagonal close packed structure of a nanowire is compatible with the often-discussed model of plastic flow of a metallic nanowire during pulling off of the electrodes.

Molecular dynamics simulations performed by Landman et al. [1] suggest that plastic flow of a nanometer-sized contact involves a series of subsequent structural transformations of a small number of atomic layers adjacent to the narrowest part of the constriction. In each transformation the neck shrinks as one atomic layer is added. Let us consider for simplicity the circular symmetric model described in Refs. 20, 21. The narrowest part of a wire is responsible for its electrical conductance.

* This form is a generalization of the expression $\lambda=$ const which was adopted in Ref. 28 and corresponds to $n=0$. 


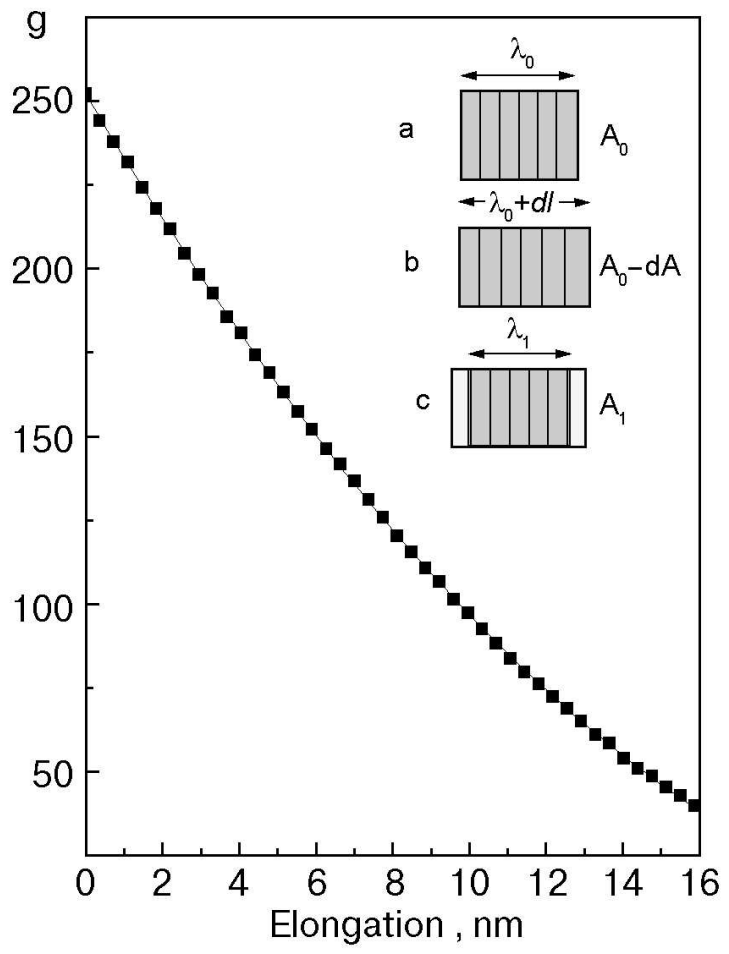

Fig. 15. Dependence of the conductance versus elongation for an averaged scan obtained by plotting positions of the atomic shell peaks in the range $30<g<250$ versus the elongation, which is calculated as $i \times h$, where $i$ is a successive integer and $h$ is the interlayer distance. The fit (curve) of the experimental data points (squares) by Eq. (11) gives an exponent $n=0.51$ and a transformation length at $g=250$ of $\lambda_{0}=13.3 \mathrm{~nm}$

Untiedt et al. propose that the part of the cylindrical constriction of the junction, which they call the transformation length $\lambda$, depends on the minimal cross section area $A$ as $\lambda=\alpha_{0} A^{n}$, where $\alpha_{0}$ is a constant and $n$ is an exponent". Depending on the indentation procedure and other parameters of the experiment (temperature, elongation rate, presence of adsorbates, etc.), $\alpha_{0}$ and $n$ may have various values.

According to [21], the process of pulling the nanowire can be represented schematically as shown in the inset of Fig. 15. Starting with a relatively thick bridge of cross section $A_{0}$ (see $(a)$ ), the length $\lambda_{0}$ first transforms elastically, which is schematically shown in $(b)$. The elastic elongation corresponds to a plateau in the conductance versus elongation curve. Next, it goes through a yielding stage, which corresponds to a jump-like shrinking of the diameter. Following Refs. 1, 20, 28, we assume that the wire relaxes by creating one more atomic layer (see Figs. 15, $b \rightarrow c$ of the inset). We take into account the discreteness of the atomic structure, which consists of close packed atomic layers with thickness $h$. Then, due to volume conservation, the atoms from a fraction $x$ of the surface layer with a transformation length $\lambda$ and a wire radius $R$, $2 \pi R x \lambda(A) h$, should be transferred to the new atomic layer along the wire axis $\pi R^{2} h$ (see Fig. 15). Equating these two quantities we obtain

$$
2 \pi R x \lambda(A) h=\pi R^{2} h, \quad \text { or } \quad \lambda(A)=R / 2 x .
$$

In this model a proportionality between $\lambda$ and $R$ exists regardless of the presence of the atomic shell effect, as was shown in Ref. 21. Taking into account that at elevated temperatures the proportionality between piezo voltage and elongation may be violated, we use instead a model to extract a scale for the elongation. We assume that the distance between the conductance peaks of the atomic-shell structure corresponds to an elongation $h=\sqrt{8 / 3} r_{0}$, equal to the distance between the close packed layers of atoms ( $r_{0}$ is the atomic radius). In this way we obtain the conductance-versus-elongation curve shown in Fig. 15. Fitting this curve to the model dependence [21]

$$
g(l)=g_{\max }\left(1-\frac{n l}{\lambda_{0}}\right)^{1 / n},
$$

where $l$ is the elongation, $g_{\max }=g(l=0)$ and $\lambda_{0}=\lambda(l=0)$, we obtain $\lambda / R \approx 3.1$. Here we start the elongation at the conductance $g_{\max }=250$, which corresponds to $R_{\max }=4.26 \mathrm{~nm}$ and $\lambda_{0}=13.3 \mathrm{~nm}$. From $\lambda \approx 3 R$ it follows that $x=1 / 6$ which perfectly correlates with hexagonal symmetry of closed packed atoms in the nanowire. This is important, because while stretching the nanowire, the crystal facets do not evaporate like in massspectroscopy, but should be built into the thinning wire, if we neglect their escape to the electrodes.

\section{Summary}

Thus, we present the evidence for two sets of magic numbers in metallic nanowires: electronic and atomic, in complete analogy with cluster physics. These two sets of oscillations in the conductance histogram compete with each other, as in cluster physics, where the transition from the electronic shell structure to the atomic shell structure is also observed for larger diameters [9]. One shellclosing effect is related to the energy of the total volume of electrons, for which the amplitude of the oscillations in the thermodynamic potential decreases as $1 / R$. The other is due to the surface energy, for which the amplitude of the oscillations is roughly constant as a function of $R$. The transi- 
tion between them depends on the parameters of experiment. The atomic-shell oscillations are observed at larger diameters (conductances) than the electronic ones, but they may overlap substantially. For $\mathrm{Li}$, and in many cases for $\mathrm{Na}$, the electronicshell structure oscillations completely dominate the spectrum.

The periodic peak structure in the histograms is only observed at temperatures well above helium temperature. Thermal energy is required in order to have sufficient mobility of the atoms allowing the structure to accommodate to the lowest free energy. Potassium has the lowest melting temperature among three alkali metals ( $\mathrm{Li}, \mathrm{Na}, \mathrm{K}$ ) studied by us. This means that at a given temperature its atoms have the highest mobility and for this metal we obtain the largest number of oscillations in the conductance histogram. From our data we cannot exclude other atomic wire arrangements, since they strongly depend on the material studied $[41,42]$. However, in the latter studies beyond a critical radius of the order of three atomic distances, the bulk lattice structure is recovered. Therefore, it is likely that the nanowires with diameters in the range of the atomic-shell structure oscillations observed here have a regular atomic stacking structure. On the other hand, the alkali metals $\mathrm{Li}$ and $\mathrm{Na}$ have low temperature martensitic phase transitions toward a close packing atomic structure and it is likely that the surface tension favors close packing in the nanowires.

Since we have studied conductance histogram for alkaline metals up to high conductances, we can claim that almost all the structure observed originates from favorable atomic configurations and not from conductance quantization, unlike the initial few peaks $(1,3,6)$ at low temperatures, where transmission measurements convincingly show degenerate quantized conductance modes [18]. However, the favorable atomic configurations are influenced by the quantization of the electronic levels, and only the atomic shell structure is a purely classical effect, but still on the atomic scale.

I. K. Y. is grateful to the Kamerlingh Onnes Laboratory of Leiden University for its hospitality and for the possibility to participate in the work presented in this paper. Special thanks to Prof. L. J. de Jongh for his interest and continuous support.

1. U. Landman, W. D. Luedtke, N. A. Burnham, and R. J. Colton, Science 248, 454 (1990)

2. B. J. van Wees, H. van Houten, C. W. J. Beenakker, J. G. Williamson, L. P. Kouwenhoven, D. van der Marel, and C. T. Foxon, Phys. Rev. Lett. 60, 848 (1988).
3. D. A. Wharam, T. J. Thornton, R. Newbury, M. Pepper, H. Ahmed, J. E. F. Frost, D. G. Hasko, D. C. Peacock, D. A. Ritchie, and G. A. C. Jones, J. Phys. C21, L209 (1988).

4. J. M. Krans, J. M. van Ruitenbeek, V. V. Fisun, I. K. Yanson, and L. J. de Jongh, Nature 375, 767 (1995).

5. A. I. Yanson, G. Rubio Bollinger, H. E. van den Brom, N.Agräit, and J. M. van Ruitenbeek, Nature 395, 783 (1998).

6. H. Ohnishi, Y. Kondo, and K. Takayanagi, Nature 395, 780 (1998).

7. W. D. Knight, K. Clemenger, W. A. de Heer, W. A. Saunders, M. Y. Chou, and M. L. Cohen, Phys. Rev. Lett. 52, 2141 (1984).

8. W. A. de Heer, Rev. Mod. Phys. 65, 611 (1993).

9. M. Brack, Rev. Mod. Phys. 65, 677 (1993).

10. T. P. Martin, Phys. Rep. 273, 199 (1996).

11. A. I. Yanson, I. K. Yanson, and J. M. Ruitenbeek, Nature 400, 1440 (1999).

12. A. I. Yanson, I. K. Yanson, and J. M. Ruitenbeek, Phys. Rev. Lett. 84, 5832 (2000).

13. A. I. Yanson, I. K. Yanson, and J. M. Ruitenbeek, to be published.

14. C. J. Muller, J. M. van Ruitenbeek, and L. J. de Jongh, Physica C191, 485 (1992).

15. E. N. Bogachek, A. N. Zagoskin, and I. O. Kulik, Sov. J. Low Temp. Phys. 16, 796 (1990).

16. J. A. Torres, J. I. Pascual, and J. J. Saenz, Phys. Rev. B49, 16581 (1994).

17. B. Ludoph and J. M. van Ruitenbeek, Phys. Rev. Lett. 82, 1530 (1999).

18. B. Ludoph and J. M. van Ruitenbeek, Phys. Rev. B61, $2273(2000)$.

19. I. K. Yanson and A. V. Khotkevich, Atlas of Point-Contact Spectra of Electron-Phonon Interaction in Metals, Naukova Dumka, Kiev (1986).

20. J. A. Torres and J. J. Saenz, Phys. Rev. Lett. 77, 2245 (1996).

21. C. Untiedt, G. Rubio, S. Vieira, and N. Agräit, Phys. Rev. B56, 2154 (1997)

22. O. Yu. Kolesnychenko, O. I. Shklyarevskii, and H. van Kempen, Rev. Sci. Instr. 70, 1442 (1999).

23. C. Z. Li, H. Sha, and N. J. Tao, Phys. Rev. B58, 6775 (1998).

24.Yu. F. Komnik, Fiz. Tver. Tela 10, 312 (1968).

25. L. J. Lewis, P. Jensen, and J. L. Barrat, Phys. Rev. B56, 2248 (1997).

26. Tables of Physical Quantities (A Handbook), I. K. Kikoin (ed.), Atomizdat, Moscow (1976).

27. C. Höppler and W. Zwerger, Phys. Rev. Lett. 80, 1792 (1998).

28. A. Stalder and U. Dürig, J. Vac. Sci. Technol. B14, 1259 (1259).

29. S. Blom, H. Olin, J. L. Costa-Krämer, N. Garsia, M. Jonson, P. A. Serena, and R. I. Shekhter, Phys. Rev. B57, 8830 (1998).

30. C. Höppler and W. Zwerger, Phys. Rev. B59, R7849 (1999).

31. J. M. van Ruitenbeek, M. H. Devoret, D. Esteve, and C. Urbina, Phys. Rev. B56, 12566 (1997).

32. E. N. Bogachek and G. A. Gogadze, Zh. Exp. Teor. Fiz. 63, 1839 (1972) [Sov. Phys. JETP 36, 973 (1973)].

33. C. A. Stafford, D. Baeriswyl, and J. Bürki, Phys. Rev. Lett. 79, 2863 (1997).

34. C. Yannouleas and U. Landman, J. Phys. Chem. B101, 5780 (1997). 
35. C. Yannouleas, E. N. Bogachek, and U. Landman, Phys. Rev. B57, 4872 (1998).

36. J. Lermé, Ch. Bordas, M. Pellarin, B. Baguenard, J. L. Vialle, and M. Broyer, Phys. Rev. B48, 12110 (1993).

37. L. D. Landau and E. M. Lifshitz, Course of Theoretical Physics, Vol. 3, Pergamon Press (1976).

38. R. Balian and C. Bloch, Ann. Phys. 69, 76 (1972).

39. J. Pedersen, S. Björnholm, J. Borggreen, C. Bréchignac, K. Hansen, T. P. Martin, and H. D. Rasmussen, Nature 353, 733 (1991).
40. R. N. Barnett and U. Landman, Nature 387, 788 (1997).

41. O. Gülseren, F. Ercolessi, and E. Tossati, Phys. Rev. Lett. 80, 3775 (1998).

42. Y. Kondo and K. Takayanagi, Science 289, 606 (2000).

43. Y. Kondo and K. Takayanagi, Phys. Rev. Lett. 79, 3455 (1997).

44. T. P. Martin, U. Näher, and H. Schaber, Chem. Phys. Lett. 199, 470 (1992). 